Swarthmore College

Works

$10-1-2001$

\title{
X-Ray Line Profiles From Parameterized Emission Within An Accelerating Stellar Wind
}

S. P. Owocki

David H. Cohen

Swarthmore College, dcohen1@swarthmore.edu

Follow this and additional works at: https://works.swarthmore.edu/fac-physics

Part of the Astrophysics and Astronomy Commons

Let us know how access to these works benefits you

\section{Recommended Citation}

S. P. Owocki and David H. Cohen. (2001). "X-Ray Line Profiles From Parameterized Emission Within An Accelerating Stellar Wind". Astrophysical Journal. Volume 559, Issue 2. 1108-1116. DOI: 10.1086/322413 https://works.swarthmore.edu/fac-physics/10

This work is brought to you for free by Swarthmore College Libraries' Works. It has been accepted for inclusion in Physics \& Astronomy Faculty Works by an authorized administrator of Works. For more information, please contact myworks@swarthmore.edu. 


\title{
X-RAY LINE PROFILES FROM PARAMETERIZED EMISSION WITHIN AN ACCELERATING STELLAR WIND
}

\author{
STANLEY P. OWOCKI \\ Bartol Research Institute, University of Delaware, 217 Sharp Laboratory, Newark, DE 19716; owocki@bartol.udel.edu \\ AND \\ DAVID H. COHEN \\ Department of Physics and Astronomy, Swarthmore College, 500 College Avenue, Swarthmore, PA 19081; dcohen1@swarthmore.edu \\ Received 2000 December 24; accepted 2001 June 1
}

\begin{abstract}
Motivated by recent detections by the $X M M$ and Chandra satellites of X-ray line emission from hot, luminous stars, we present synthetic line profiles for X-rays emitted within parameterized models of a hot-star wind. The X-ray line emission is taken to occur at a sharply defined comoving-frame resonance wavelength, which is Doppler-shifted by a stellar wind outflow parameterized by a " $\beta$ " velocity law, $v(r)=v_{\infty}\left(1-R_{*} / r\right)^{\beta}$. Above some initial onset radius $R_{o}$ for X-ray emission, the radial variation of the emission filling factor is assumed to decline as a power law in radius, $f(r) \sim r^{-q}$. The computed emission profiles also account for continuum absorption within the wind, with the overall strength characterized by a cumulative optical depth $\tau_{*}$. In terms of a wavelength shift from line center scaled in units of the wind terminal speed $v_{\infty}$, we present normalized X-ray line profiles for various combinations of the parameters $\beta, \tau_{*}, q$, and $R_{o}$ and also including the effect of instrumental and/or macroturbulent broadening as characterized by a Gaussian with a parameterized width $\sigma$. We discuss the implications for interpreting observed hot-star X-ray spectra, with emphasis on signatures for discriminating between "coronal" and "wind-shock" scenarios. In particular, we note that in profiles observed so far the substantial amount of emission longward of line center will be difficult to reconcile with the expected attenuation by the wind and stellar core in either a wind-shock or coronal model.

Subject headings: line: profiles — radiative transfer — shock waves — stars: early-type stars: mass loss $-\mathrm{X}$-rays: stars

On-line material: color figures
\end{abstract}

\section{INTRODUCTION}

Over the past two decades, orbiting X-ray observatories from Einstein to ASCA have shown that both hot and cool stars are moderately strong sources of soft X-rays at energies between 0.1 and $2 \mathrm{keV}$. For cooler, late-type stars, the scaling of X-ray luminosity with, e.g., stellar rotation, suggests a solar-type origin, with surface magnetic loops confining hot, X-ray-emitting material within nearly static circumstellar coronae. For hotter, early-type stars, the $\mathrm{X}$-ray luminosity has no discernible dependence on rotation but instead scales nearly linearly with bolometric luminosity $L_{x} \approx 10^{-7} L_{\mathrm{Bol}}$ (Long \& White 1980; Pallavicini et al. 1981; Chlebowski, Harnden, \& Sciortino 1989), suggesting a different emission mechanism, perhaps related to the high-density, radiatively driven stellar wind observed from such stars.

Nonetheless, an ongoing question has remained whether such hot-star X-ray emission originates from coronal-like processes occurring in nearly static regions near the stellar surface (Cassinelli \& Olson 1979; Waldron 1984; Cohen, Cassinelli, \& MacFarlane 1997) or rather in the highly supersonic wind outflow, perhaps from shocks arising from intrinsic instabilities in the radiative driving mechanism (Lucy \& White 1980; Lucy 1982; Owocki, Castor, \& Rybicki 1988; Feldmeier et al. 1997a; Feldmeier, Puls, \& Pauldrach 1997b; Owocki \& Cohen 1999). Based on the observed strong winds in hot stars (see, e.g., Snow \& Morton 1976), a key discriminant has been the apparent general lack of soft X-ray absorption, which, along with optical and UV spectral constraints, imply limits to the extent, temperature, and fractional contribution of the total X-ray output from a base corona (Cassinelli, Olson, \& Stalio 1978; Nordsieck, Cassinelli, \& Anderson 1981; Cassinelli \& Swank 1983; Baade \& Lucy 1987; MacFarlane et al. 1993; Cohen et al. 1996). This limited inferred absorption thus is generally seen as evidence for emission arising from lower density regions at large radii, which is in general agreement with a wind-shock model.

In the context of EUV spectroscopy, MacFarlane et al. (1991) presented models of emission-line profiles, from both a coronal source and an expanding shell, affected by transfer through a stellar wind. These authors demonstrated that emission-line profiles are a key discriminant of the source location. However, because of the small throughput and modest resolution of the Extreme Ultraviolet Explorer $(E U V E)$ spectrometers, combined with the extreme opacity of the interstellar medium in this bandpass, emission lines from only one hot star $(\epsilon \mathrm{CMa})$ were observed with the $E U V E$. The only high signal-to-noise ratio line seen in this EUV spectrum was the He II Ly $\alpha$ line, which was observed to be broadened by several hundred kilometers per second, lending credence to models that postulate a wind origin for the hot plasma in OB stars (Cassinelli et al. 1995).

It is only recently, however, that orbiting X-ray observatories like $X M M$ and Chandra have been able to provide the first detections of spectrally resolved X-ray emission 
lines from hot, luminous stars (Kahn et al. 2001; Schulz et al. 2000; Waldron \& Cassinelli 2001; Cassinelli et al. 2001). Through the broadened profiles of these resolved X-ray emission lines, these data provide a new, key diagnostic for inferring whether the X-ray emission originates from a nearly static corona or from an expanding stellar wind outflow. A key goal of the present paper is to provide a firm basis for interpreting such X-ray emission-line spectra.

Specifically, we describe below the method (§ 2) and results $(\S 3)$ for computations of synthetic line profiles arising from parameterized forms of X-ray emission in an expanding stellar wind. In comparison to recent work by Ignace (2001), who derived analytic forms for X-ray emission profiles for the case of constant expansion velocity, our analysis takes into account a variation of velocity $v(r)$ in radius $r$, as parameterized by the so-called $\beta$-law form $v(r)=v_{\infty}\left(1-R_{*} / r\right)^{\beta}$, where $R_{*}$ is the stellar radius and $v_{\infty}$ is the wind terminal speed. As in Ignace (2001), the overall level of attenuation is characterized by an integral optical depth $\tau_{*}$, and the X-ray emission filling factor is assumed to decline outward as a power law in radius, $f(r) \sim r^{-q}$. However, we also allow for this emission to begin at a specified onset radius $R_{o}$ that can be set at or above the surface radius $R_{*}$. We first present scaled emission profiles for various combinations of the four parameters $\beta, \tau_{*}, q$, and $R_{o} / R_{*}(\S 3.1)$. For selected cases intended to represent roughly the competing models of a "base coronal" emission versus instability-generated "wind-shock" emission, we next compute synthetic profiles that are broadened by the estimated instrumental resolution for emission lines observed by $X M M$ and Chandra ( $\$ 3.2)$. Finally, we discuss $(\S 4)$ how various features of the synthetic profiles are influenced by properties of the emitting material and outline prospects for future work in interpreting hot-star X-ray spectra.

\section{COMPUTING X-RAY LINE EMISSION FROM A SPHERICAL STELLAR WIND}

Extended monitoring observations by $\mathrm{X}$-ray missions like ROSAT (Berghöfer \& Schmitt 1994) have shown that for most early-type stars, the X-ray emission is remarkably constant, with little or no evidence of rotational modulation and with overall variations at or below the $1 \%$ level. This suggests that the hot, X-ray-emitting material must be well distributed within the circumstellar volume, whether in a narrow, near-surface corona or as numerous $[n>(1 /$ $\left.0.01)^{2}=10^{4}\right]$, small elements of shock-heated gas embedded in the much cooler, UV line-driven, ambient stellar wind. Here we model any such complex structure in terms of a spherically symmetric, smooth distribution of X-ray line emission, with emissivity $\eta_{\lambda}(r, \mu)$ at an observer's wavelength $\lambda$ along direction $\cos \mu$ from a radius $r$. The resulting $\mathrm{X}$-ray luminosity spectrum $L_{\lambda}$ is given by integrals of the emission over direction and radius, attenuated by continuum absorption within the medium,

$$
L_{\lambda}=8 \pi^{2} \int_{-1}^{1} d \mu \int_{R_{*}}^{\infty} d r r^{2} \eta_{\lambda}(\mu, r) e^{-\tau(\mu, r)} .
$$

\subsection{Optical Depth Integration}

The absorption optical depth $\tau(\mu, r)$ can be most easily evaluated by converting to ray coordinates $z \equiv \mu r$ and $p \equiv\left(1-\mu^{2}\right)^{1 / 2}$ and then integrating over distance $z$ for each ray with impact parameter $p$,

$$
t(p, z)=\int_{z}^{\infty} \kappa \rho\left(r^{\prime}\right) d z^{\prime},
$$

where $\rho\left(r^{\prime}\right)$ is the mass density at radius $r^{\prime} \equiv\left(p^{2}+z^{\prime 2}\right)^{1 / 2}$. We assume here that the X-ray absorption cross section per unit mass $\kappa$ is a fixed constant that is independent of wavelength $\lambda$ within the line and radius $r$ within the wind. For emission at a given $r$ along direction $\cos \mu$, the optical depth attenuation in equation (1) can then be obtained through the simple substitution

$$
\tau(\mu, r)=t\left(\sqrt{1-\mu^{2}} r, \mu r\right) .
$$

For simplicity, we consider here a steady state wind with velocity given by the " $\beta$-law" form $w(r) \equiv v(r) / v_{\infty}=(1$ $\left.-R_{*} / r\right)^{\beta}$. Taking account also of the occultation by the stellar core of radius $R_{*}$, we then write

$$
t(p, z)= \begin{cases}\infty, & p \leq R_{*} ; z \leq \sqrt{R_{*}^{2}-p^{2}}, \\ \tau_{*} \int_{z}^{\infty} \frac{R_{*} d z^{\prime}}{r^{\prime 2}\left(1-R_{*} / r^{\prime}\right)^{\beta}}, & \text { otherwise }\end{cases}
$$

Here we have used the mass-loss rate $\dot{M} \equiv 4 \pi \rho v r^{2}$ to define a characteristic wind optical depth $\tau_{*} \equiv \kappa \dot{M} / 4 \pi v_{\infty} R_{*}$. Note that in a wind with $\beta=0$ and thus a constant velocity $v=v_{\infty}$, the radial $(p=0)$ optical depth at radius $r$ would be given simply by $t(0, r)=\tau_{*} R_{*} / r$. Thus, in such a constantvelocity wind, $\tau_{*}$ would be the radial optical depth at the surface radius $R_{*}$, while $R_{1}=\tau_{*} R_{*}$ would be the radius of the unit radial optical depth.

For noninteger $\beta$, the optical depth integral (eq. [4]) must generally be done by numerical quadrature, but for general integer values, e.g., $\beta=0,1,2$, or 3 , it is still possible to obtain closed form analytic expressions for this optical depth, although the expressions can be quite cumbersome to write down explicitly (see also Ignace 2001). In the present work, these analytic forms are derived and evaluated using the mathematical analysis software mathematica. To provide a specific example, we cite here the analytic result for the canonical case of $\beta=1$, for which the integral in equation (4) becomes

$$
\begin{aligned}
& \frac{t(p, z)}{\tau_{*}}= \\
& \left(\frac{\arctan \left\{\left[\left(z^{\prime} / R_{*}\right) / r^{\prime}\right] / \sqrt{p^{2}-R_{*}}\right\}+\arctan \left(z / \sqrt{p^{2}-R_{*}}\right)}{\sqrt{p^{2}-1}}\right)_{z^{\prime}=z}^{z^{\prime} \rightarrow \infty} .
\end{aligned}
$$

This expression actually applies under the full general conditions cited in equation (4), although some care must be taken in its evaluation for the cases with $p \leq 1$.

\subsection{Line Emission Integration}

Let us next consider the line emissivity, which we take here to be of the form

$$
\eta_{\lambda}(r, \mu)=C \rho^{2}(r) f(r) \delta\left\{\lambda-\lambda_{o}[1-\mu v(r) / c]\right\},
$$


where $C$ is an emission normalization constant, $\lambda_{o}$ is the rest wavelength for the line emission, and $f(r)$ represents a volume filling factor for X-ray emission. Note that the radial dependence of this filling factor may reflect variations in the temperature distribution of the plasma as well as variations in the total emission measure.

Equation (6) simply assumes that the emission is an arbitrarily sharp function centered on the local, Dopplershifted, comoving-frame wavelength $\lambda_{o}[1-\mu v(r) / c]$ and so ignores all broadening (e.g., natural, Stark, thermal, turbulent). For Doppler shifts associated with either thermal or small-scale "microturbulent" motions, the $\delta$ function should be replaced with a finite-width emission profile, but generally, such microscopic motions can be expected to be no greater than the gas sound speed, which for X-ray-emitting plasma of temperature $T_{\mathrm{X}} \sim 10^{7} \mathrm{~K}$, is on the order of a few times $100 \mathrm{~km} \mathrm{~s}^{-1}$. As such, the associated broadening should be relatively small compared to that expected from the directed motion of the wind outflow, which has a much larger characteristic speed $v_{\infty}>1000 \mathrm{~km}$ $\mathrm{s}^{-1}$. Moreover, as discussed in $\S 3.2$, for larger scale, "macroturbulent" motions, the effects can be taken into account a posteriori by incorporating them with the general smearing of the observed line profiles expected from the limited spectral resolution of the X-ray detector.

For convenience, let us define a scaled wavelength measured from line center in units of the wind terminal speed $v_{\infty}$, i.e., $x \equiv\left(\lambda / \lambda_{o}-1\right) c / v_{\infty}$. In terms of this scaled wavelength, the integrals (eq. [1]) for the luminosity spectrum then become

$$
\begin{aligned}
L_{x}= & 8 \pi^{2} C \int_{-1}^{1} d \mu \int_{R_{*}}^{\infty} d r r^{2} f(r) \rho^{2}(r) e^{-\tau[\mu, r]} \\
& \times \delta[x+\mu w(r)],
\end{aligned}
$$

where we have used the convention $L_{x} d x=L_{\lambda} d \lambda$. Integrating the Dirac $\delta$-function over direction cosine $\mu$, we then find that the wavelength-dependent X-ray luminosity can be evaluated from the single radial integral,

$$
\begin{aligned}
L_{x}= & \frac{C \dot{M}^{2}}{2 v_{\infty}^{2}} \int_{R_{*}}^{\infty} d r H[w(r)-|x|] \\
& \times \frac{f(r)}{r^{2} w^{3}(r)} e^{-\tau[-x / w(r), r]},
\end{aligned}
$$

where $H$ is the Heaviside (aka unit step) function, with $H(s)=1$ if $s \geq 0$ and zero otherwise.

Figure 1 plots the optical depth $\tau[-x / w(r), r]$ versus scaled wavelength $x$ at selected radii $r$ in models with various $\beta$. For each radius, there is a critical, positive wavelength $x$ at which the optical depth increases steeply, representing the strong attenuation of the red wing emission by the dense inner wind and stellar core. Such core occultation makes the integrand in equation (9) effectively zero for all $r$, such that $\left(1-R_{*}\right)^{\beta}\left(1-R_{*}^{2}\right)^{1 / 2}<x$. For blue wing wavelengths $x<0$, the termination of the curves indicates the maximum blueshift allowed by the velocity at the relevant radius.
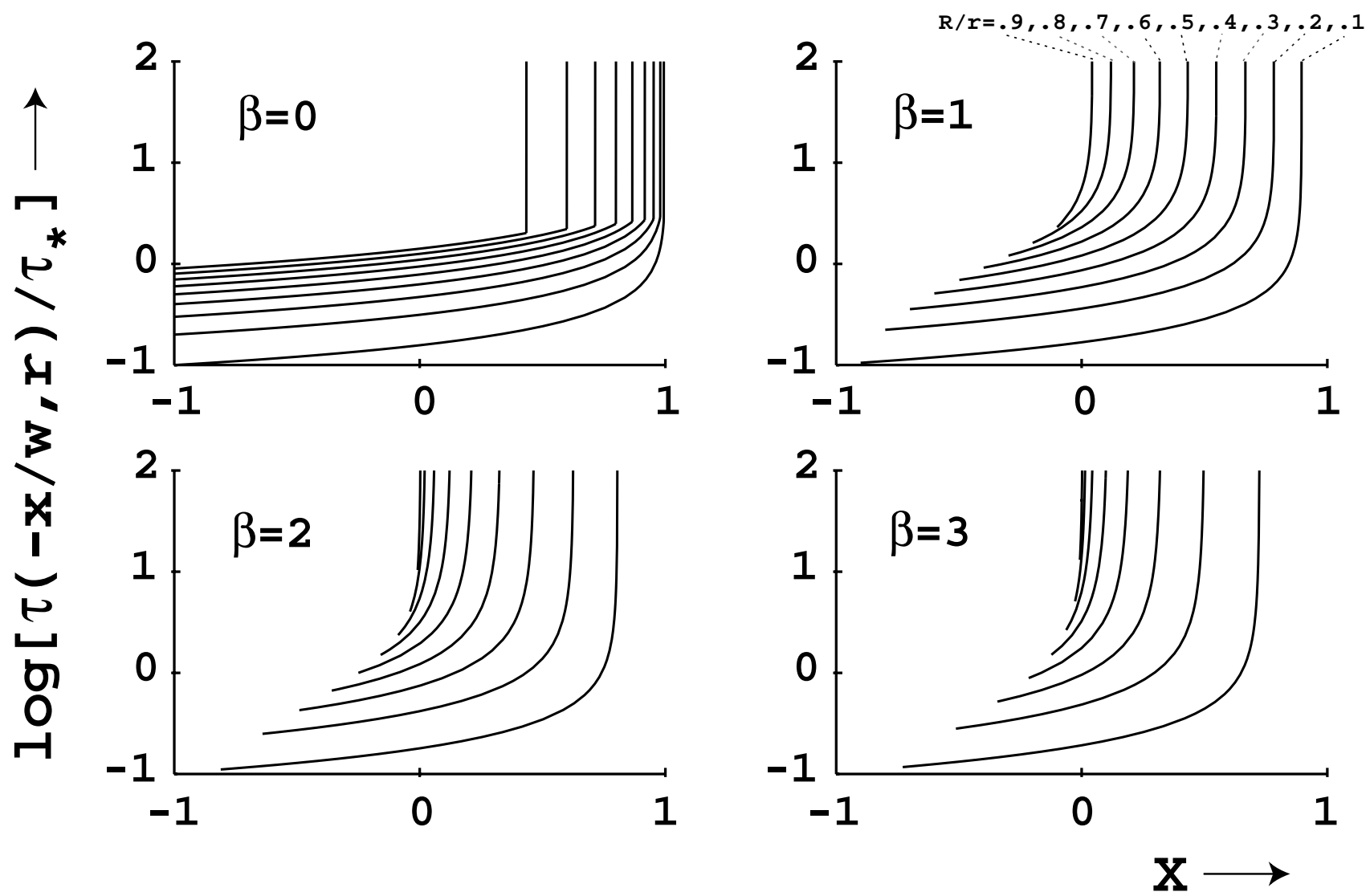

Fig. 1.-The $\log$ of the scaled optical depth $\tau[-x / w(r), r] / \tau_{*}$ vs. the scaled wavelength $x$ at selected radii $r$ in models with $\beta=0,1,2$, and 3. These contours effectively show the degree of wind attenuation as a function of wavelength for a source at a given radius. [See the electronic edition of the Journal for a color version of this figure.] 
Ignace (2001) has recently derived completely analytic results for the $\mathrm{X}$-ray profiles assuming a power-law emission factor $f(r) \sim r^{-q}$ and constant outflow velocity $\beta=0$ [implying $w(r)=1]$. As noted above, for the somewhat more general case of nonzero, but integer values of $\beta$, the optical depth integral (eq. [4]) can still be done analytically, but in general, the radial integral (eq. [8]) must be evaluated by numerical quadrature. For this numerical integration, it is convenient (following Ignace 2001) to define an inverse radius coordinate $u \equiv R_{*} / r$, yielding

$$
L_{x}=\frac{C \dot{M}^{2}}{2 v_{\infty}^{2} R_{*}} \int_{0}^{u_{x}} d u \frac{f\left(R_{*} / u\right)}{(1-u)^{3 \beta}} e^{-\tau\left[-x /(1-u)^{\beta}, R_{*} / u\right]},
$$

where $u_{x} \equiv 1-|x|^{1 / \beta}$.

\section{RESULTS FOR NORMALIZED LINE PROFILES FROM POWER-LAW X-RAY EMISSION}

For any chosen model for the spatial variation of emission $f(r)$, equation (9) can be readily evaluated through a single numerical integration. As a specific illustration, we now present results for the example case of an emission factor with the power-law form $f(r)=f_{*} u^{q}$ for radii $r \equiv$ $R_{*} / u>R_{o} \equiv R_{*} / u_{o}$ and zero otherwise. This cutoff implies a slight redefinition to the upper integral bound in equation (9),

$$
u_{x} \equiv \min \left(u_{o}, 1-|x|^{1 / \beta}\right) .
$$

To focus on the general form of X-ray line emission, let us define a normalized emission profile,

$$
l_{x} \equiv \frac{L_{x}}{\max _{x}\left(L_{x}\right)},
$$

which simply sets the profile maximum to have a value of unity. This effectively removes the dependence on the several free parameters (e.g., $f_{*}, \dot{M}, v_{\infty}$, etc.) that affect the overall emission normalization. The form of the resulting $\mathrm{X}$-ray line profile $l_{x}$ versus scaled wavelength $x$ thus depends on four free parameters, viz., $\beta, \tau_{*}, q$, and $R_{o} / R_{*}$. The effects of wind attenuation and other model parameters on the overall X-ray emission level have been addressed in a broadband context by Owocki \& Cohen (1999), but in focussing here on line profile shapes, we defer to future work any analysis of the overall emission-line strength.

\subsection{Synthetic Emission-Line Profiles for Various Model Parameters}

Figure 2 presents synthetic emission profiles for various combinations of these parameters, all plotted versus the scaled wavelength $x$ over the range $-1<x<1$. For comparison, Figure 3 shows the flat-topped profiles for corresponding cases with optically thin emission, $\tau_{*}=0.01$. Some notable general properties and trends are as follows:

1. The profiles seem most sensitive to $\tau_{*}$ and $R_{o}$, with $\beta$ and $q$ having a comparatively modest influence.

2. The emission peaks are always blueward of line center (i.e., at $x<0$ ), with increasing blueshift for increasing $\tau_{*}$ and/or increasing $R_{o}$.

3. The overall line width likewise increases with increasing $\tau_{*}$ and/or $\boldsymbol{R}_{o}$. The narrowest lines occur when both $\tau_{*}$ and $R_{o}$ are near unity. Large $\tau_{*}$ with low $R_{o}$ tends to give blueshifted "peaked" forms, while large $R_{o}$ tends to be bluetilted "flat-top" forms.

4. The blue-to-red slope near line center is always negative. For low $\tau_{*}$, it varies from very steep for low $R_{o}$ to nearly flat for large $R_{o}$. For large $\tau_{*}$, it has a more intermediate steepness for all $R_{o}$.

\subsection{Broadened Profiles for Coronal versus Wind-Shock Scenarios}

For this simple parameterized model, the profiles presented above represent the expected intrinsic line emission, with the only broadening associated with the directed, large-scale wind outflow. In practice, observed line profiles can be further broadened, by both the limited spectral resolution of the detector as well as by Doppler shifting from additional, random motions of the emitting gas. As already noted in $\S 2.2$, for small-scale thermal or "microturbulent" motions, the associated broadening can be expected to be no larger than the gas sound speed, which, for $T_{x} \sim 10^{7} \mathrm{~K}$ plasma, is on the order of a few $100 \mathrm{~km} \mathrm{~s}^{-1}$. Since this is much less than the $v_{\infty}>1000 \mathrm{~km} \mathrm{~s}^{-1}$ characteristic of the directed wind outflow, it should have a comparatively minor effect on line profiles arising from the outer wind. For the inner wind or more static "coronal" models, larger scale turbulent broadening, which might, in principle, have a larger characteristic speed, can still be accounted for a posteriori, together with instrumental broadening arising from the limited spectral resolution of the detectors used in the $X M M$ and Chandra satellites. For simplicity, we assume here that both macroturbulent and instrumental broadening can be characterized by convolving a simple Gaussian kernel function over the intrinsic line profile. As such, a single parameterized width $\sigma$, defined here in units of the wind terminal speed $v_{\infty}$, represents the combined effects of both any macroturbulent motion and instrumental broadening, with $\sigma \equiv\left(\sigma_{\text {turb }}^{2}+\sigma_{\text {instr }}^{2}\right)^{1 / 2}$.

Let us now consider examples of such broadened line profiles. To keep the parameter range tractable, we identify specific parameter subsets to represent general conditions in the two distinct scenarios that have often been invoked in modeling hot-star X-ray emission, namely, the "coronal" (see, e.g., Waldron 1984) versus wind-shock scenarios (see, e.g., Feldmeier et al. 1997b; Owocki \& Cohen 1999). The coronal scenario envisions $\mathrm{X}$-ray emission as coming mostly from a relatively narrow, nearly static layer near the stellar surface; we represent this here with the parameter values $q=5, \beta=3$, and $R_{o}=R_{*}$. The wind-shock scenario generally envisions $\mathrm{X}$-ray production arising from instability-generated shocks that form in the acceleration region $r=1-3 R_{*}$ of the star's line-driven wind, with eventual slow decay of the shocks in the outer wind; we represent this here with the parameter values $R_{o}=1.5 R_{*}$, $\beta=1$, and $q=0.5$. For both scenarios, we again consider the effect of various optical depth parameters $\tau_{*}$.

Figure 4 compares results for the coronal (left panel) versus wind-shock (right panel) scenario. In characterizing results, we shall refer to profiles with small versus large $\sigma$ as representing good/high versus coarse/low resolution. Here some notable general properties and trends include the following:

1. At the best resolution $(\sigma=0.1)$, the coronal models are, as expected, notably narrower than the wind models. 


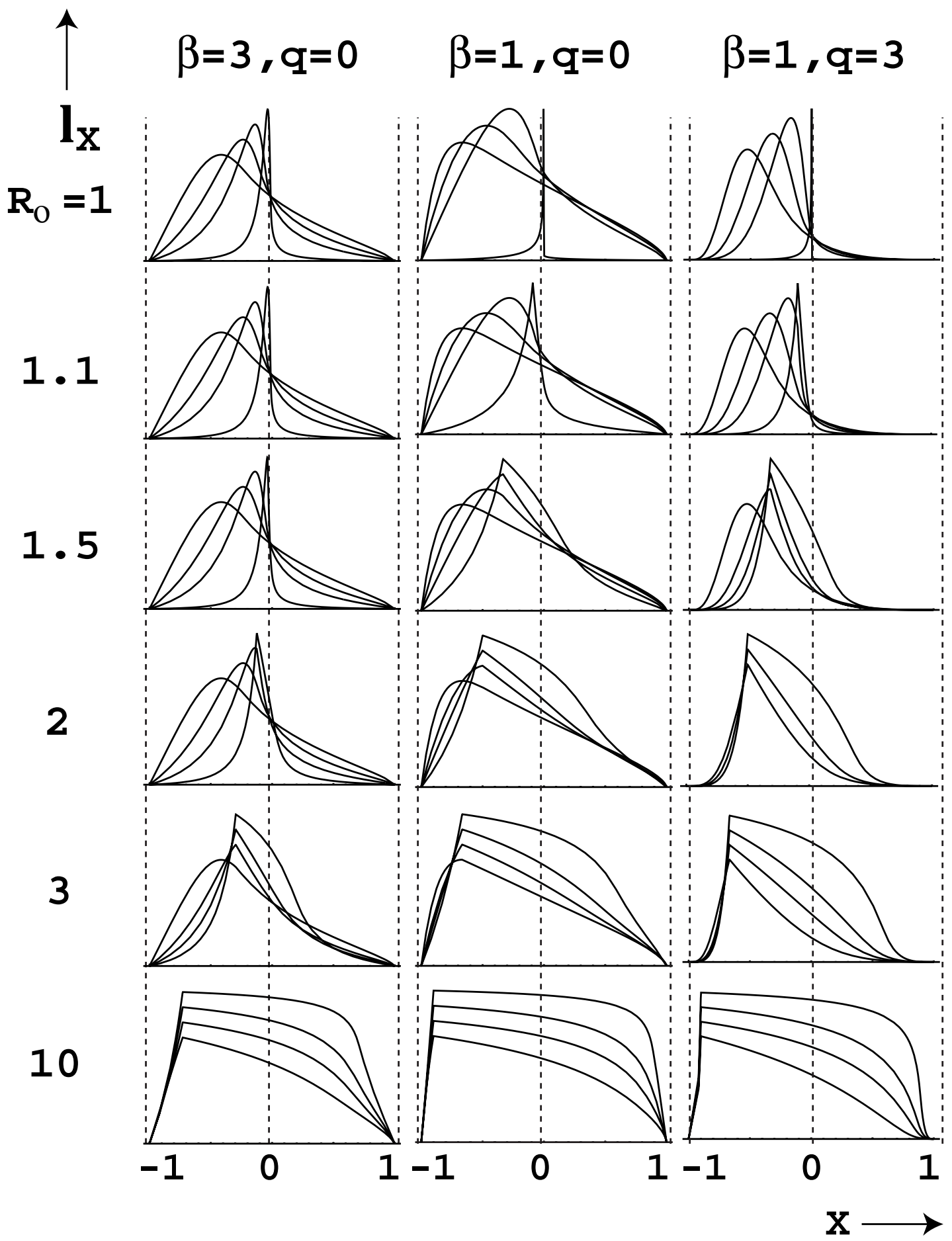

FIG. 2. - Scaled emission profile $l_{x}$ vs. the scaled wavelength $x$ plotted for various parameter combinations. In each box, profiles for optical depths $\tau_{*}=1$, 3,5 , and 10 are overplotted, with the various curves distinguished by having their peak values scaled by factors of $1,0.9,0.8$, and 0.7 , respectively. The six rows represent, from the top to bottom, cases with initial emission radii of $R_{o} / R_{*}=1,1.1,1.5,2,3$, and 10 . The central of the three columns represents a "standard" case with $\beta=1$ and $q=0$, while the columns to each side illustrate cases that differ from this standard by setting $\beta=3$ (left) or $q=3$ (right). [See the electronic edition of the Journal for a color version of this figure.]

2. The wind-shock models are generally more blueshifted than the coronal models.

3. At the coarsest resolution $(\sigma=0.5)$, all profiles have similar width but still generally differ in overall blueshift.

4. For the optically thin case, the emission is nearly symmetric about line center. For other cases, the emission peaks become more blueshifted with increasing optical depth $\tau_{*}$.
(The coronal models with larger $\tau_{*}$ may not be relevant, however, since wind absorption would greatly reduce the overall strength of any observable emission.)

The typical spectral resolution for Chandra is around $\lambda / \Delta \lambda \sim 1000-1500$. For a characteristic wind terminal speed of $v_{\infty} \sim 2000-3000$, this implies a typical broadening 

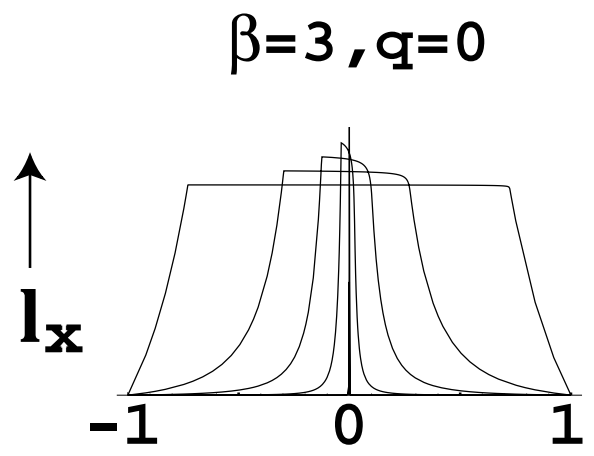
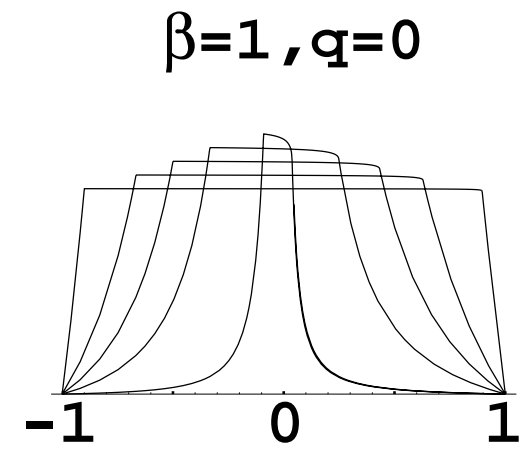
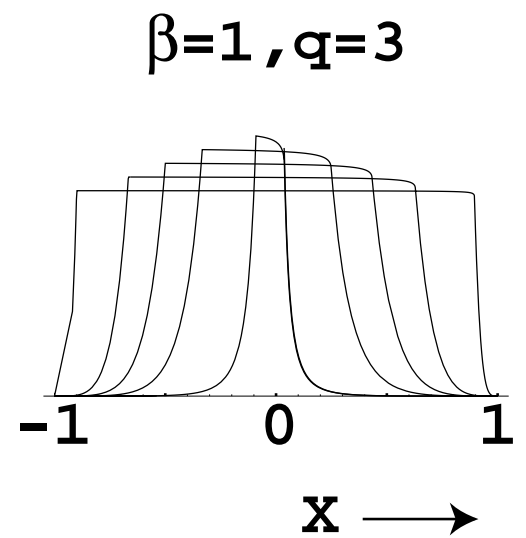

Fig. 3.-Flat-topped emission profiles for the optically thin case $\tau_{*}=0.01$. The left, center, and right images correspond to the same wind parameters as in Fig. 2, but the five overplots in each image now represent cases with initial emission radii at $R_{o} / R_{*}=1.1,1.5,2,3$, and 10 , with the various curves now distinguished by having their peak values scaled by factors of $1,0.95,0.9,0.85$, and 0.8 , respectively. [See the electronic edition of the Journal for a color version of this figure.]

parameter of $\sigma \sim 0.1$. For $X M M$, the spectral broadening is comparable, although slightly larger. Clearly, at such high resolutions, it should be possible to place strong constraints on the site of X-ray emission and the degree of wind attenuation as well as on the characteristic speeds of directed versus turbulent motions of the emitting plasma.

\section{DISCUSSION}

The parameterized emission models here provide a good basis for interpreting X-ray line profile observations made possible by the new generation of orbiting X-ray telescopes. While detailed, quantitative analyses must await the general availability of specific data sets, it is already clear from the few hot-star X-ray spectra published thus far that there are some noteworthy challenges for modeling the associated $\mathrm{X}$-ray emission.

For example, temperature-sensitive line ratio diagnostics provide constraints on the magnitude of shocks in dynamical models. Generally, instability-generated wind-shock models tend to be dominated by moderate shock strengths, $\Delta v \sim 100-400 \mathrm{~km} \mathrm{~s}^{-1}$, that can only heat material up to a maximum of $\sim 10^{7} \mathrm{~K}$ (Feldmeier et al. 1997a). In those stars with much higher inferred temperatures, for example $\theta^{1}$ Ori C with $T>5 \times 10^{7} \mathrm{~K}$ (Schulz et al. 2000), there is a need to produce stronger shocks. One possibility is for wind outflow to be channeled by magnetic loops (Babel \& Montmerle 1997). In this case, wind material accelerated up opposite footpoints of a closed loop can be forced to collide near the loop top, in principle yielding shocks with velocity jumps on the order of $\Delta v \sim 1000 \mathrm{~km} \mathrm{~s}^{-1}$, which is a substantial fraction of the wind terminal speed.

Another key diagnostic stems from the forbidden-tointercombination $(f / i)$ ratios of helium-like emission lines, which can provide a strong constraint on the location of the $\mathrm{X}$-ray emission. In the case of the solar corona, such ratios are nominally interpreted as density diagnostics (Gabriel \& Jordan 1969), but because the strong UV radiation of hot stars can generally dominate over electron collisions in the destruction of forbidden-line upper levels, in such stars the $f / i$ provides instead a constraint on the strength of the UV intensity (Blumenthal, Drake, \& Tucker 1972; Mewe \& Schrijver 1978). With appropriate atmospheric modeling of the relevant part of UV continuum, any inferred dilution of the radiation thus provides a diagnostic of the proximity of the X-ray emission to the stellar surface. For $X M M$ observations of $\zeta$ Pup (Kahn et al. 2001), initial interpretations suggest formation well away from the stellar surface $(r>2$ $R_{*}$ ), consistent with a wind-shock origin, with, moreover, a general trend toward larger radii for lower energy lines, as would be expected from the larger optical depths and thus larger unit optical depth radii for such lines. For Chandra observations of $\zeta$ Ori Waldron \& Cassinelli (2001), the inferred formation radii are also generally away from the star $\left(r \approx 2-10 R_{*}\right)$ but with one stage (Si XIII) suggesting a formation very close to the surface $\left(r<1.1 R_{*}\right)$. However, in this latter case, the relevant UV flux for destruction of the forbidden upper levels lies at energies above the Lyman edge, and so any error in atmospheric modeling of the Lyman jump could still alter this inferred formation radius. Chandra observations of $\zeta$ Pup (Cassinelli et al. 2001) show similar results, with most inferred formation radii in the range $r \approx 1.5-10 R_{*}$, with trends again consistent with inferred unit optical depth radii, but again with one stage (in this case, $\mathrm{S} \mathrm{Xv}$ ) implying formation quite close to the surface $\left(r<1.25 R_{*}\right)$. Of course, such $(f / i)$ constraints on source location must eventually be considered in conjunction with additional constraints, such as those provided by analysis of line profiles.

Indeed, in the context of this paper, the most notable new diagnostic provided by recent X-ray observations lies in the form of the line profiles. For some initial results, these have some quite puzzling characteristics. For example, in the $X M M$ spectrum of $\zeta$ Pup (Kahn et al. 2001), the lines all exhibit a similar, significant broadening but with halfwidths $\sim 1000-1500 \mathrm{~km} \mathrm{~s}^{-1}$ that are still somewhat lower than the inferred wind terminal speed $v_{\infty} \gtrsim 2000 \mathrm{~km} \mathrm{~s}^{-1}$. The typical profiles have a rounded, Gaussian form that is quite distinct from, e.g., the flat-topped profiles expected for optically thin emission from a nearly constant velocity expansion. Moreover, the profiles show only modest asymmetry, with the peak intensity only slightly blueshifted, by $\sim 0-400 \mathrm{~km} \mathrm{~s}^{-1}$ relative to line center, and the red wing only slightly flatter and more extended than the blue wing. Recent, somewhat higher spectral resolution observations by Chandra do show the greater shift and asymmetry expected from optically thick wind emission for $\zeta$ Pup 


\section{Coronal}

$\mathbf{R}_{\mathbf{0}}=\mathbf{1}, \mathbf{q}=5, \beta=3$
Wind-shock

$$
R_{0}=1.5, q=0.5, \beta=1
$$

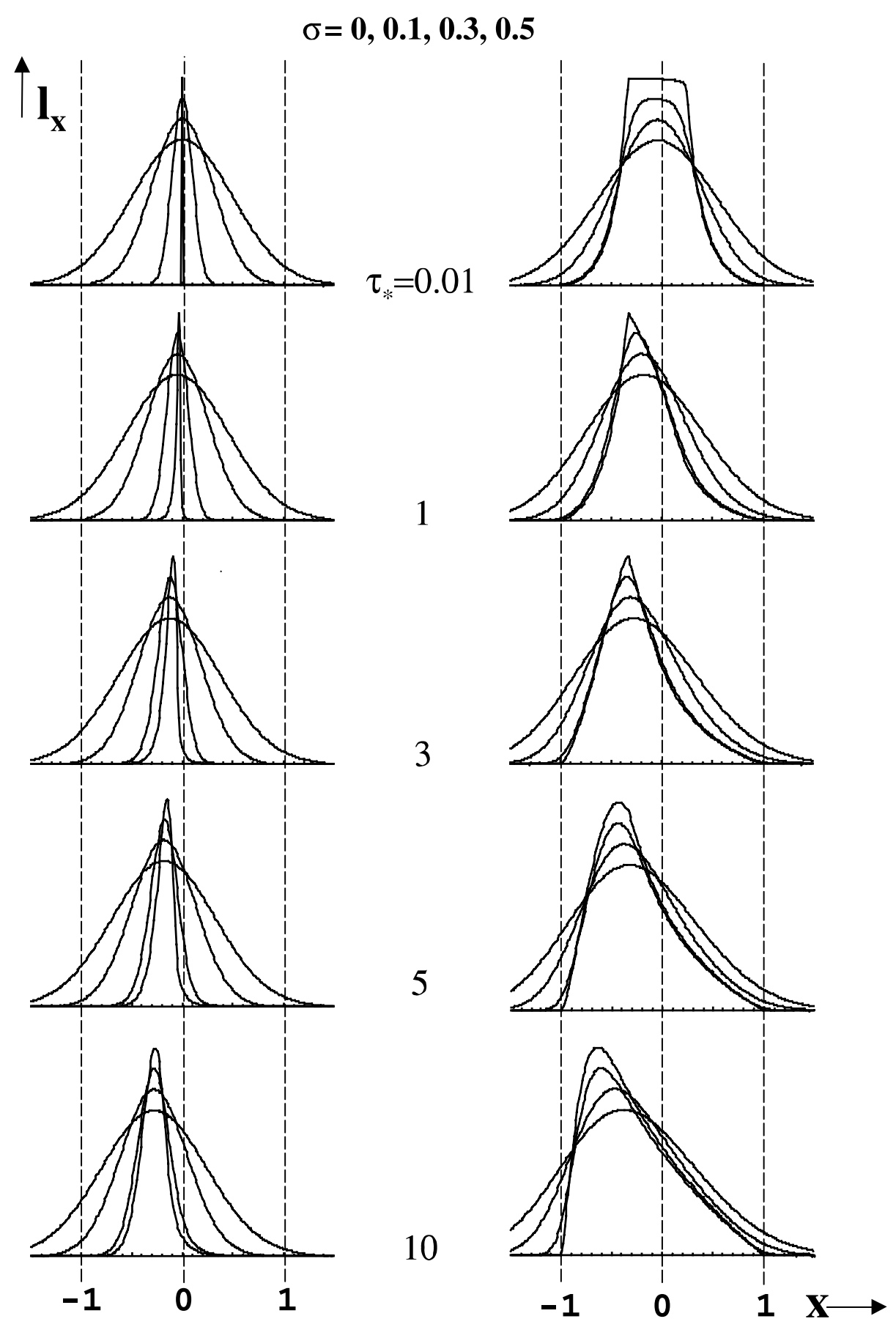

Fig. 4. - As in Fig. 2, scaled emission profile $l_{x}$ vs. the scaled wavelength $x$, but now comparing results for a coronal (left) vs. wind-shock (right) scenario. The four overplots now represent profiles with broadening parameters set to $\sigma=0,0.1,0.3$, and 0.5 , again with the overall emission scaled by factor of $1,0.9$, 0.8 , and 0.7 , respectively. The five rows now represent (from the top) cases with optical depth parameters set to $\tau_{*}=0.01,1,3,5$, and 10 . [See the electronic edition of the Journal for a color version of this figure.]

(Cassinelli et al. 2001), but in Chandra observations of the O9.7 Ib star $\zeta$ Ori, the profiles are again very broad (halfwidths of $\sim 1000 \mathrm{~km} \mathrm{~s}^{-1}$ ) but nearly symmetric (Waldron \& Cassinelli 2001).

Such profiles with substantial broadening, but little asymmetry and blueshift, are difficult to reconcile with a simple wind outflow model that includes attenuation by the wind and stellar core, both of which should preferentially attenuate X-rays from the backside hemisphere, and thus, in a wind or corona with some outflow, preferentially reduce the red side emission. In fact, from detailed modeling constrained by ROSAT observations, Hillier et al. (1993) have argued that the wind of $\zeta$ Pup should be optically thick to 1 $\mathrm{keV} \mathrm{X}$-rays at $10 R_{*}$, with the radius of optical depth unity 
being even greater for softer $\mathrm{X}$-rays $\left(20 R_{*}\right.$ at $0.6 \mathrm{keV}$ and still $2 R_{*}$ at $2 \mathrm{keV}$ ).

Even for a base corona with some overall outflow velocity and no overlying wind attenuation, occultation by the stellar core should still produce line profiles that are skewed toward the blue. A coronal model with little or no net outflow could explain the observed profile symmetry, but matching the observed line widths would then require the coronal material to have extremely large "turbulent" motions. For example, line widths in the Chandra observations of $\zeta$ Ori (Waldron \& Cassinelli 2001) require velocity dispersions on the order of $1000 \mathrm{~km} \mathrm{~s}^{-1}$, and some of the lines observed by Chandra in $\theta^{1}$ Ori C (Schulz et al. 2000) are even broader than this and yet apparently still nearly symmetric. If the lack of red wing attenuation is interpreted to mean that redshifted emission does not arise from wind outflow from the backside hemisphere, then this suggests that material in front of the star must be flowing downward at velocities of $1000 \mathrm{~km} \mathrm{~s}^{-1}$. Since this is greater than the surface escape speed, it cannot be the result of gravitational infall but instead would imply that some mechanism, e.g., perhaps magnetic reconnection (Waldron \& Cassinelli 2001), must actively accelerate roughly equally strong upflow and downflow with speeds of $1000 \mathrm{~km} \mathrm{~s}^{-1}$ ! Moreover, such random motions should inevitably lead to highspeed gas collisions and their associated shock heating. For shocks with velocity jumps of order $1000 \mathrm{~km} \mathrm{~s}^{-1}$, the associated postshock temperature should be on the order of $10^{8} \mathrm{~K}$, implying generally harder X-rays and higher ionization stages than are typically observed. In this context, these broad but symmetric profiles would indeed seem to represent a strong challenge for theoretical models.

On the other hand, our synthesized line profiles from wind models do seem capable of producing relatively broad, yet symmetric and unshifted profiles, but only in the nearly complete absence of wind attenuation and with emission originating far from the occultation of the stellar core. To test the viability of this alternative, observed line profiles should be interpreted in conjunction with other diagnostics, like the $f / i$ ratio, that can constrain the radii of X-ray emission and with an accurate account of wind absorption, as computed through opacity calculations for the observationally inferred wind mass-loss rate.

Any wind-shock model with significant wind attenuation is expected to be strongly blueshifted and quite asymmetric (see Fig. 4). Of all the line profiles discussed in the first group of $X M M$ and Chandra papers, the observations of $\zeta$ Pup provide the best evidence for the blueshifts and asymmetry expected for optically thick wind emission (Kahn et al. 2001; Cassinelli et al. 2001). For example, Figure 3 of
Cassinelli et al. (2001) shows five of six lines with blueward shifts and asymmetry similar to profiles calculated here for wind-shock models with substantial optical depth, e.g., $\tau_{*} \sim 5$. At least for this canonical O-type star, these profiles seems to provide strong evidence of a wind origin for most of the observed X-rays.

In summary, systematic fitting of the line profiles will be necessary to place more quantitative constraints on the spatial distribution of hot plasma and the degree of wind attenuation in the observed $\mathrm{O}$ stars, but some initial qualitative inferences are already possible. For some cases, the implied minimal wind attenuation combined with some significant bulk motion is puzzling, both in the context of coronal X-ray emission and in the context of the strong radiation-driven winds that are known to exist on these stars from UV, optical, IR, and radio observations. One possible solution to the wind attenuation problem might be found in clumping. A "porous" wind might allow many of the X-rays to stream through unattenuated yet might also lead to significant UV absorption if the interclump wind medium still has sufficient column density to produce optically thick UV lines. (The UV line transition probabilities are significantly larger than the bound-free $\mathrm{X}$-ray cross sections.) An alternative is that the mass-loss rates of $O$ stars may have been overestimated. It does not seem likely, however, that the winds are dense and smooth but transparent to X-rays since that would require levels of ionization in the wind that would not allow for efficient radiation driving, and, if the winds are indeed optically thin, then to discriminate between coronal and wind models will require a careful analysis of the shifts and asymmetries observed in the X-ray line profiles.

Finally, although we developed our analysis with hot stars in mind, it could be extended to other X-ray sources in which lines are emitted within a nearly symmetric expanding medium that may also have substantial continuum opacity. This could include nova winds, planetary nebulae, active galactic nuclei, and X-ray binaries.

We thank R. Ignace for providing a preprint of his paper on analytic X-ray profiles and also for his detailed comments on this manuscript. We also thank K. Gayley and A. Ud-Doula for helpful comments on our analysis and J. Cassinelli and J. MacFarlane for many useful insights regarding the X-ray spectra of O stars. Finally, we thank the referee, M. Corcoran, for helpful comments and suggestions on the submitted manuscript. This research was supported in part by NASA grant NAG 5-3530 to the Bartol Research Institute at the University of Delaware.

\section{REFERENCES}

Baade, D., \& Lucy, L. 1987, A\&A, 178, 213

Babel, J., \& Montmerle, T. 1997, ApJ, 485, L29

Berghöfer, T. W., \& Schmitt, J. H. M. M. 1994, Ap\&SS, 221, 309

Blumenthal, G. R., Drake, G. W. F., \& Tucker, W. H. 1972, ApJ, 172, 205

Cassinelli, J. P., et al. 1995, ApJ, 438, 932

Cassinelli, J. P., Miller, N. A., Waldron, W. L., MacFarlane, J. J., \& Cohen, D. H. 2001, ApJ, 554, L 55

Cassinelli, J. P., \& Olson, G. L. 1979, ApJ, 229, 304

Cassinelli, J. P., Olson, G. L., \& Stalio, R. 1978, ApJ, 220, 573

Cassinelli, J. P., \& Swank, J. H. 1983, ApJ, 271, 681

Chlebowski, T., Harnden, F. R., Jr., \& Sciortino, S. 1989, ApJ, 341, 427

Cohen, D. H., Cassinelli, J. P. \& MacFarlane, J. J. 1997, ApJ, 487, 867

Cohen, D. H., Cooper, R. G., MacFarlane, J. J., Owocki, S. P., Cassinelli, J. P., \& Wang, P. 1996, ApJ, 460, 506

Feldmeier, A., Kudritzki, R.-P., Palsa, R., Pauldrach, A. W. A., \& Puls, J. 1997a, A\&A, 320, 899
Feldmeier, A., Puls, J., \& Pauldrach, A. W. A. 1997b, A\&A, 322, 878

Gabriel, A. H., \& Jordan, C. 1969, MNRAS, 145, 241

Hillier, D. J., Kudritzki, R. P., Pauldrach, A. W., Baade, D., Cassinelli, J. P., Puls, J., \& Schmitt, J. H. M. M. 1993, A\&A, 276, 117

Ignace, R. 2001, ApJ, 549, L119

Kahn, S. M., Leutenegger, M. A., Cotam, J., Rauw, G., Vreux, J.-M., den Boggende, A. J. F., Mewe, R., \& Güdel, M. 2001, A\&A, 365, L312

Long, K. S., \& White, R. L. 1980, ApJ, 239, L65

Lucy, L. 1982, ApJ, 255, 286

Lucy, L. B., \& White, R. L. 1980, ApJ, 241, 300

Macarlane, J. J., Cassinelli, J. P., Welsh, B. Y., Vedder, P. W., Vallerga, J. V., \& Waldron, W. L. 1991, ApJ, 380, 564

MacFarlane, J. J., Waldron, W. L., Corcoran, M. F., Wolff, M. J., Wang, P., \& Cassinelli, J. P. 1993, ApJ, 419, 813

Mewe R., \& Schrijver, J. 1978, A\&A, 65, 99

Nordsieck, K. H., Cassinelli, J. P., \& Anderson, C. M. 1981, ApJ, 248, 678 
Owocki, S. P., Castor, J. I., \& Rybicki, G. B. 1988, ApJ, 335, 914

Owocki, S. P., \& Cohen, D. H. 1999, ApJ, 520, 833

Pallavicini, R., Golub, L., Rosner, R., Vaiana, G. S., Ayres, T., \& Linsky,

Snow, T. P., Jr., \& Morton, D. C. 1976, ApJS, 32, 429

Waldron, W. L. 1984, ApJ, 282, 256

J. L. 1981, ApJ, 248, 279

Schulz, N. S., Canizares, C. R., Huenemoerder, D., \& Lee, J. C. 2000, ApJ, 545, L135

Note added in proof.-R. Ignace \& K. G. Gayley (ApJ, submitted [2001]) have recently noted that including optically thick line effects can yield much more rounded line profiles in wind models with a constant velocity. We have since included such effects in our models with wind acceleration and confirm that these will tend to round off the square profiles produced with large X-ray onset radius $R_{o}$; however, the effects on X-ray emission from the wind acceleration region are less pronounced. Further details will be reported in a future paper that will focus on detailed fitting of observed X-ray line profiles. 